\title{
EFFECTS OF THERMAL RADIATION AND VISCOUS DISSIPATION ON POWELL- EYRING NANOFLUID WITH VARIABLE THICKNESS
}

\author{
D. VIDYANADHA BABU ${ }^{1} \&$ M. SURYANARAYANA REDDY ${ }^{2}$ \\ ${ }^{I}$ Department of mathematics, Priyadarshani College of Engineering, Sullurpet, Nellore Dt, A.P., India \\ ${ }^{2}$ Department of mathematics, JNTUA College of engineering, Pulivendula, A.P, India
}

\begin{abstract}
A steady boundary layer flow of Powell- Eyring nanofluid past a stretching sheet with variable thickness in the presence of thermal radiation and viscous dissipation is studied numerically. The model is used for the nanofluid incorporates the effects of Brownian motion and thermophoresis. The suitable transformations are applied to convert the governing partial differential equations into a set of nonlinear coupled ordinary differential equations. Runge-Kutta-based shooting technique is employed to yield the numerical solutions for the model. The obtained results for the velocity, temperature and concentration are analyzed graphically for several physical parameters. It is found that an increment in wall thickness parameter results in decrease of velocity, temperature and concentration profiles. Further, in tabular form the numerical values are given for the local skin friction coefficient, local Nusselt number and Sherwood number. A remarkable agreement is noticed by comparing the present results with the results reported in the literature as a special case.
\end{abstract}

KEYWORDS: Thermal Radiation, Powell - Eyring Fluid, Heat Viscous Dissipation \& MHD

Received: Jul 07, 2017; Accepted: Jul 31, 2017; Published: Aug 09, 2017; Paper Id.: IJMPERDAUG201739

\section{INTRODUCTION}

Viscous boundary layer flow due to a stretching/shrinking sheet is of significant importance due to its vast applications. Aerodynamic extrusion of plastic sheets, glass fiber production, paper production, heat treated materials travelling between a feed roll and a wind-up roll, cooling of an infinite metallic plate in a cooling bath and manufacturing of polymeric sheets are some examples for practical applications of non-Newtonian fluid flow over a stretching/shrinking surface. The quality of the final product depends on the rate of heat transfer at the stretching surface. This stretching/shrinking may not necessarily be linear. It may be quadratic, power-law, exponential and so on. Bilal Asharf et.al. [1] Studied the three dimensional boundary layer flow of Eyrng-Powell nanofluid by convectively heated exponentially stretching sheet. Hayat et.al. [2] Investigated the axisymmetric Powell-Eyring fluid flow with convective boundary condition. Javed et.at. [3] Analyzed the boundary layer flow of an Eyring Powell non-Newtonian fluid over a stretching sheet. Hayat et.al. [4] Studied the effect of MHD boundary layer flow of Powell-Eyring nanofluid over a non-linear stretching sheet with variable thickness. Nazar et.al. [5] Investigated the mixed convection boundary layer flow an isothermal horizontal circular cylinder embedded in a porous medium filled with a nanofluid for both cases of a heated and cooled cylinder.

Solar energy is probably the most suitable source of renewable energy that can meet the current energy requirements. The energy obtained from nature in the form of solar radiations can be directly transformed into heat and electricity. The idea of using small particles to collect solar energy was first investigated by Hunt [6] in the 
1970s. Researchers concluded that with the addition of nanoparticles in the base fluids, heat transfer and the solar collection processes can be improved. Masuda et al. [7] discussed the alteration of thermal conductivity and viscosity by dispersing ultra-fine particles in the liquid. Choi and Eastman [8] were the first to introduce the terminology of nanofluids when they experimentally discovered an effective way of controlling heat transfer rate using nanoparticles. Buongiorno [9] developed the nonhomogeneous equilibrium mathematical model for convective transport of nanofluids. He concluded that Brownian motion and thermophoretic diffusion of nanoparticles are the most important mechanisms for the abnormal convective heat transfer enhancement. The relevant processes are briefly described in [10-12]. Investigations in the nanofluid flows have received remarkable popularity in research community in last couple of decades primarily due to their variety of applications in power generation, in transportation where nanofluid may be utilized in vehicles as coolant, shock absorber, fuel additives etc., in cooling and heating problems which may involve the use of nanofluids for cooling of microchips in computer processors, in improving performance efficiency of refrigerant/air-conditioners etc. and in biomedical applications in which magnetic nanoparticles may be used in medicine, cancer therapy and tumor analysis. Recently the researchers have proposed the idea of using solar collector based nanofluids for optimal utilization of solar energy radiation [13, 14]. Buongiorno and $\mathrm{Hu}[15]$ discussed the heat transfer enhancement via nanoparticles for nuclear reactor application. Huminic and Huminic [16] showed that use of nanofluids in heat exchangers has advantage in the energy efficiency and it leads to better system performance. Ramzan et.al. [17] Discussed effects radiation and MHD on Powell-Eyring nanofluid over a stretching cylinder with chemical reaction. Ullah et.al. [18] Studied theoretically the influence of thermal radiation and MHD on natural convective flow of casson nanofluid over a non linearly stretching sheet. Mustafa et.al. [19] Runge-Kutta fourth -fifth order method with shooting technique to explore the steady boundary layer flow of nanofluid past a vertical plate with nonlinear radiation. Numerical solution for steady boundary layer flow of dusty fluid over a radiating stretching surface embedded in a thermally stratified porous medium in the presence of uniform heat source was studied by Gireesha et.al. [20]. Recently, Ramzan et.al. [21] Analyzed the radiative Jeffery nanofluid with convective heat and mass conditions.

To the best of the author's knowledge, there seems no existing document about steady boundary layer flow of Powell- Eyring nanofluid past a stretching sheet with variable thickness in the presence of thermal radiation and heat viscous dissipation. Hence the aim of the present investigation is to examine the steady boundary layer flow of PowellEyring nanofluid past a stretching sheet with variable thickness in the presence of thermal radiation and viscous dissipation.

\section{MATHEMATICAL FORMULATION}

Radiative powell-Eyring nanofluid over an impermeable nonlinear heated stretching sheet with variable thickness is considered. An incompressible fluid is selected electrically conducting. A non-uniform magnetic field $B(x)=B_{0}(x+b)^{\frac{n-1}{2}}$ is imposed transverse to the stretching sheet. Magnetic Reynolds number is chosen small. Induced magnetic and electric fields are not accounted. Brownian and thermophoresis in the nanofluid are considered. Newly developed condition for mass flux is imposed. The fluid formation is such that the $x$-axis is presumed along the sheet while $y$-axis is transverse to it. The temperature of the sheet is different from that of the ambient medium. The fluid viscosity is assumed to vary with the temperature while the other fluid properties are assumed constant.

The stretching surface has the nonlinear velocity $U_{w}=U_{0}(x+b)^{n}$ where $U_{0}$ is the reference velocity and b is 
the dimensional constant. Further it is assumed that sheet at $y=A(x+b)^{\frac{1-n}{2}}$ is not flat (where $n$ is the velocity power index and $A$ is assumed very small so that the sheet retain adequately thin). We also noticed that for $n=1$ the problem reduces to a flat sheet. The governing expressions for considered flow are given by:

$$
\begin{aligned}
& \frac{\partial u}{\partial x}+\frac{\partial v}{\partial y}=0 \\
& u \frac{\partial u}{\partial x}+v \frac{\partial u}{\partial y}=\left(v+\frac{1}{\rho \beta d}\right) \frac{\partial^{2} u}{\partial y^{2}}-\frac{1}{2 \rho \beta d^{3}}\left(\frac{\partial u}{\partial y}\right)^{2} \frac{\partial^{2} u}{\partial y^{2}}-\frac{\sigma B^{2}(x)}{\rho} u \\
& u \frac{\partial T}{\partial x}+v \frac{\partial T}{\partial y}=\alpha_{f} \frac{\partial^{2} T}{\partial y^{2}}+\tau\left(D_{B} \frac{\partial T}{\partial y} \frac{\partial C}{\partial y}+\frac{D_{T}}{T_{\infty}}\left(\frac{\partial T}{\partial y}\right)^{2}\right)-\frac{1}{(\rho c)_{f}} \frac{\partial q_{r}}{\partial y}+\frac{v}{c_{f}}\left(\frac{\partial u}{\partial y}\right)^{2} \\
& u \frac{\partial C}{\partial x}+v \frac{\partial C}{\partial y}=D_{B} \frac{\partial^{2} C}{\partial y^{2}}+\frac{D_{T}}{T_{\infty}}\left(\frac{\partial^{2} T}{\partial y^{2}}\right)
\end{aligned}
$$

With boundary conditions

$$
\begin{aligned}
& u=U_{w}(x)=U_{0}(x+b)^{n}, v=0, T=T_{w}, C=C_{w} \quad \text { at } \quad y=A(x+b)^{\frac{1-n}{2}} \\
& u \rightarrow 0, v \rightarrow 0, T \rightarrow T_{\infty}, \quad C \rightarrow C_{\infty} \text { as } y \rightarrow \infty
\end{aligned}
$$

Here $\mathrm{u}$ and $\mathrm{v}$ are the corresponding velocity components parallel to $x$ - and $y$-directions respectively, $\mu$ the dynamic viscosity, $v=\frac{\mu}{\rho}$ designates the kinematic viscosity, $\rho$ the fluid density, $d$ and $\beta$ are the material liquid parameters of Powell-Eyring model, $k$ the thermal conductivity, $\alpha_{n f}\left(=\frac{k}{(\rho c)_{f}}\right)$ the thermal diffusivity of liquid, $\tau$ the ratio of the heat capacity of liquid of the nanoparticles material to the effective heat capacity of the base fluid, $D_{B}$ indicates the Brownian diffusion coefficient, $D_{T}$ represents the thermophoretic diffusion, $T$ the temperature of the fluid, $C$ the nanoparticles concentration, $T_{w}$ and $T_{\infty}$ are the sheet and ambient fluid temperatures and $C_{\infty}$ the ambient fluid nanoparticles concentration. 


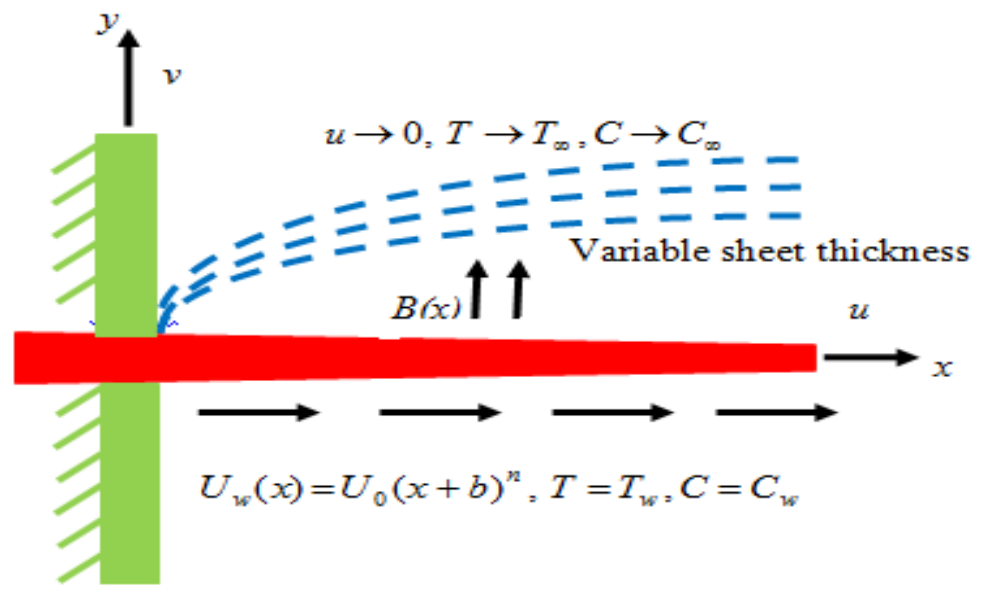

Figure 1: Physical Model and Coordinate System

Using the Rossel and approximations, the radiative heat flux is given by

$$
q_{r}=-\frac{4 \sigma^{*}}{3 K^{*}} \frac{\partial T^{4}}{\partial z}
$$

Where, $\sigma^{*}$ and $K^{*}$ are, respectively, the Stephan - Boltzman constant and the mean absorption coefficient. Assume that the temperature differences within the flow are small such that $T^{4}$ in a Taylor series about $T_{\infty}$ and neglecting higher order terms. We get

$$
T^{4} \cong 4 T_{\infty} T-3 T_{\infty}^{4}
$$

Substituting eq.'s (7) and (8) in eq. (3). We have

$$
u \frac{\partial T}{\partial x}+v \frac{\partial T}{\partial y}=\left(\alpha_{f}+\frac{16 T_{\infty} \sigma^{*}}{3 K^{*}(\rho c)_{f}}\right) \frac{\partial^{2} T}{\partial y^{2}}+\tau\left(D_{B} \frac{\partial T}{\partial y} \frac{\partial C}{\partial y}+\frac{D_{T}}{T_{\infty}}\left(\frac{\partial T}{\partial y}\right)^{2}\right)+\frac{v}{c_{f}}\left(\frac{\partial u}{\partial y}\right)^{2}
$$

Transformations are expressed as follows:

$$
\begin{aligned}
& u=U_{0}(x+b)^{n} F^{\prime}(\eta), v=-\sqrt{\left(\frac{n+1}{2}\right) v U_{0}(x+b)^{n-1}}\left[F(\eta)+\eta F^{\prime}(\eta)\left(\frac{n-1}{n+1}\right)\right] \\
& \eta=y \sqrt{\left(\frac{n+1}{2}\right) \frac{U_{0}(x+b)^{n-1}}{v}}, \Theta(\eta)=\frac{T-T_{\infty}}{T_{w}-T_{\infty}}, \Phi(\eta)=\frac{C-C_{\infty}}{C_{w}-C_{\infty}}
\end{aligned}
$$

Incompressibility condition is satisfied identically and Eqs.(2), (4)-(6) and (9) take the following forms

$$
\begin{aligned}
& (1+N) F^{\prime \prime \prime}+F F^{\prime \prime}-\left(\frac{2 n}{n+1}\right) F^{\prime 2}-N\left(\frac{n+1}{2}\right) \lambda F^{\prime \prime 2} F^{\prime \prime \prime}-\left(\frac{2}{n+1}\right) M F^{\prime}=0 \\
& \left(\frac{1+N r}{\operatorname{Pr}}\right) \Theta^{\prime \prime}+F \Theta^{\prime}+N^{\prime} b \Theta^{\prime} \Phi^{\prime}+N t \Theta^{\prime 2}+E c f^{\prime \prime 2}=0
\end{aligned}
$$




$$
\begin{aligned}
& \Phi^{\prime \prime}+\operatorname{Pr} \operatorname{LeF} \Phi^{\prime}+\left(\frac{N t}{N b}\right) \Theta^{\prime \prime}=0 \\
& F(\alpha)=\alpha\left(\frac{1-n}{1+n}\right), F^{\prime}(\alpha)=1, \Theta(\alpha)=1, \Phi(\alpha)=1 \\
& F^{\prime}(\infty) \rightarrow 0, \quad \Theta(\infty) \rightarrow 0, \quad \Phi(\infty) \rightarrow 0
\end{aligned}
$$

In the above expressions primes designate differentiation with respect to $\eta$. Where $\alpha=A \sqrt{\left(\frac{n+1}{2}\right) \frac{U_{0}}{v}}$ is the wall thickness parameter and $\eta=\alpha=A \sqrt{\left(\frac{n+1}{2}\right) \frac{U_{0}}{v}}$ shows the plate surface. Upon using

$$
F(\eta)=f(\eta-\alpha)=f(\xi), \Theta(\eta)=\theta(\eta-\alpha)=\theta(\xi), \Phi(\eta)=\phi(\eta-\alpha)=\phi(\xi)
$$

Eqs.(11)-(15) yield (See fig.1).

$$
\begin{aligned}
& (1+N) f^{\prime \prime \prime}+f f^{\prime \prime}-\left(\frac{2 n}{n+1}\right) f^{\prime 2}-N\left(\frac{n+1}{2}\right) \lambda f^{\prime \prime 2} f^{\prime \prime \prime}-\left(\frac{2}{n+1}\right) M f^{\prime}=0 \\
& \frac{1+N r}{\operatorname{Pr}} \theta^{\prime \prime}+f \theta^{\prime}+N b \theta^{\prime} \phi^{\prime}+N t \theta^{\prime 2}+E c f^{\prime \prime 2}=0 \\
& \phi^{\prime \prime}+\operatorname{Pr} \operatorname{Lef} \phi^{\prime}+\left(\frac{N t}{N b}\right) \theta^{\prime \prime}=0 \\
& f(0)=\alpha\left(\frac{1-n}{1+n}\right), f^{\prime}(0)=1, \theta(0)=1, \phi(0)=1 \\
& f^{\prime}(\infty)=0, \theta(\infty)=0, \phi(\infty)=0
\end{aligned}
$$

where $N$ and $\lambda$ are the fluid parameters, $M$ represents the magnetic parameter, $N b$ the Brownian motion parameter , Pr the Prfzandtl number, $N t$ the thermophoresis parameter, Le presents Lewis number, $N r$ the radiation parameter, $E c$ the Eckert number and prime indicates differentiation with respect to $\xi$. The non-dimensional parameters are

$$
\begin{aligned}
& N=\frac{1}{d \beta \mu}, \lambda=\frac{U_{0}^{3}}{4 d^{2} v}(x+b)^{3 n-1}, M=\frac{\sigma B_{0}^{2}}{\rho U_{0}}, \operatorname{Pr}=\frac{v}{\alpha_{f}}, N b=\tau \frac{D_{B}\left(C_{w}-C_{\infty}\right)}{v} \\
& N t=\tau \frac{D_{T}\left(T_{w}-T_{\infty}\right)}{v T_{\infty}}, L e=\frac{\alpha_{f}}{D_{B}}, N r=\frac{16 \sigma^{*} T_{\infty}^{3}}{k K^{*}}, E c=\frac{U_{w}}{c_{f}\left(T_{w}-T_{\infty}\right)}
\end{aligned}
$$

Surface drag coefficient and surface heat transfer are expressed as follows: 


$$
\begin{aligned}
& C_{f}=\frac{2 \tau_{w}}{\rho_{f} U_{w}^{2}}, N u_{x}=\frac{(x+b) q_{w}}{k\left(T_{w}-T_{\infty}\right)} \\
& \tau_{w}=\left(\left(\mu_{n f}+\frac{1}{\beta d}\right) \frac{\partial u}{\partial y}-\frac{1}{6 \beta d^{3}}\left(\frac{\partial u}{\partial y}\right)^{3}\right)_{y=A(x+b)^{\frac{1-n}{2}}} \\
& q_{w}=-k\left(\frac{\partial T}{\partial y}\right)_{y=A(x+b)^{\frac{1-n}{2}}}
\end{aligned}
$$

In dimensionless form we have [37, 38]:

$$
\begin{aligned}
& C_{f} \operatorname{Re}_{x}^{1 / 2}=\sqrt{2(n+1)}\left((1+N) f^{\prime \prime}(0)-\frac{(n+1)}{2} \frac{N \lambda}{3}\left(f^{\prime \prime \prime}(0)\right)^{3}\right) \\
& N \mu_{x} \operatorname{Re}_{x}^{-1 / 2}=-\sqrt{\frac{n+1}{2}} \theta^{\prime}(0)
\end{aligned}
$$

Where $\operatorname{Re}_{x}$ designates the local Reynolds number presented by $\frac{U_{w}(x+b)}{v}$.

\section{SOLUTION OF THE PROBLEM}

The governing equations for the present problem are transformed into a set of coupled nonlinear differential equations by applying similarity transformation. The equations (16) - (18) together with the boundary conditions (19) (20) are integrated numerically by using Runge-Kutta-Gill method along with the shooting technique. This method is concisely outlined as below:

$$
\begin{aligned}
& y_{1}=f, y_{2}=f^{\prime}, y_{3}=f^{\prime \prime}, y_{4}=\theta, y_{5}=\theta^{\prime}, y_{6}=\phi, y_{7}=\phi^{\prime} \\
& y_{3}^{\prime}=\frac{1}{1+N-\lambda N\left(\frac{n+1}{2}\right) y_{3}^{2}}\left(-y_{1} y_{3}+\left(\frac{2 n}{n+1}\right) y_{2}^{2}+\left(\frac{2}{n+1}\right) M y_{2}\right) \\
& y_{5}^{\prime}=-\left(\frac{\operatorname{Pr}}{1+R}\right)\left(y_{1} y_{5}+N b y_{5} y_{7}+N t y_{5}^{2}+E c y_{3}^{2}\right) \\
& y_{7}^{\prime}=-L e \operatorname{Pr} y_{1} y_{7}-\frac{N t}{N b}\left[-\left(\frac{P r}{1+R}\right)\left(y_{1} y_{5}+N b y_{5} y_{7}+N t y_{5}^{2}+E c y_{3}^{2}\right)\right]
\end{aligned}
$$

The boundary conditions are transformed as follows:

$$
\begin{aligned}
& y_{1}(0)=\alpha\left(\frac{1-n}{1+n}\right), y_{2}(0)=1, y_{4}(0)=1, y_{6}(0)=1 \\
& y_{2}(\infty)=0, y_{4}(\infty)=0, y_{6}(\infty)=0
\end{aligned}
$$

In order to carry out the step by step integration for the equations (16) - (18), Gill's procedures have been used 
(Ralston and Wilf [23]). To start the integration it is necessary to provide all the values of $y_{1}, y_{2}, y_{3}, y_{4}, y_{5}, y_{6}$ at $\eta=0$ from which point, the forward integration has been carried out but from the boundary conditions it is seen that the values of $y_{3}, y_{4}, y_{7}$ are not known. So, we are to provide such values of $y_{3}, y_{4}, y_{7}$ along with the known values of the other function at $\eta=0$ as would satisfy the boundary conditions as $\eta \rightarrow \infty$ to a prescribed accuracy after step by step integrations are performed. Since the values of $y_{3}, y_{4}, y_{7}$ which are supplied are merely rough values, some corrections have to be made in these values in order that the boundary conditions to $\eta \rightarrow \infty$ are satisfied. These corrections in the values of $y_{3}, y_{4}, y_{7}$ are taken care of by a self-iterative procedure which can for convenience be called corrective procedure.

\section{RESULTS AND DISCUSSIONS}

In this section, we have presented the physical behavior of the velocity, temperature and concentration profiles for different physical parameters such as magnetic parameter $M$, fluid parameters $\lambda, N$, wall thickness parameter $\alpha$, Prandtl number Pr, Eckert number $E c$, thermophoresis parameter $N t$, Brownian motion parameter $N b$ and thermal radiation parameter $R$. we are solving the system of coupled nonlinear equations by using well known method Runge-Kutta-Gill method along with the shooting technique.

Figure 2 portrays velocity field for varied values of magnetic parameter $M$ and fluid parameters $N$. It is observed that velocity profiles reduces with an increase in magnetic parameter $M$, physically, presented results occurs in the conductivity fluid and create a resistive type force i.e. Lorentz force on the fluid in the boundary layer, which fall on the velocity of the fluid. In the presence of fluid parameter, it is noteworthy that the velocity profile depicts an increment with increasing $N$. The behavior of $M$ and $N$ on the temperature and concentration is shown in figs.3 and 4 respectively. Temperature and nano concentration profiles show increasing behaviour for higher values of $M$, whereas decreasing behaviour for $N$. An increment in $M$ produces higher Lorentz force (resistive force) which has the characteristic to convert some thermal energy into heat energy. Therefore the temperature and concentration profile increases. Furthermore, the opposite behaviour was observed for the effect of $N$. Physically, it justifies that higher value of $N$ produce a reduction in the fluid viscosity. Therefore, less heat is produced due to frictional force. Hence, the temperature and concentration distribution decreases.

Figure 5 displays that the wall thickness parameter $\alpha$ decreases velocity profile of the fluid physically, increasing the value of $\alpha$ decrease the flow velocity, because under the variable wall thickness, not all the pulling force of the stretching sheet can be transmitted to the fluid causing a decrease for the friction between the fluid layers. Effects of the thermophoresis parameter $N t$ and Brownian motion parameter $N b$ on temperature and nano concentration distributions are shown in figures 6 and 7. It is comprehended that temperature and concentration fields are increasing functions of thermophoretic parameter. In fact, small particles are pulled towards cold surface from hot one. Eventually, temperature of the fluid increases which results in higher temperature and nano concentration profiles. From these figures, it also analyzed that temperature and concentration distributions show increasing and decreasing behaviour for higher values of Brownian motion parameter with upsurge in values of Brownian motion parameter an increase in fluid particles random motion is observed that eventually produce more heat. So, we observe increasing temperature field and decreasing concentration distribution. Figure 8 shows that the effects of thermal radiation parameter and Eckert number on temperature profiles. $R=$ $O$ shows that no radiation on the fluid layer and $E c=O$ represents the no viscous dissipation on the fluid layer. The 
temperature profile increases with an increase in the thermal radiation and Eckert number.

Furthermore, from the figure 9 and 10, it is observed that an increase in the Prandtl number results in decreasing the heat and mass transfer profiles. The reason is that increasing values of Prandtl number is equivalent to decreasing the thermal conductivities, and therefore heat is able to diffuse away from the heated sheet more rapidly. Hence, in the case of increasing Prandtl number the boundary layer is thinner and the heat transfer is reduced. The effect of Lewis number on concentration profile is presented in figure 11. It is observed that concentration profiles decreases with increase in $L e$. Figures 12 and 13 depicted for the effects of thermophoresis parameter and Brownian motion parameter on local Nusselt number and local Sherwood number, respectively. From these figures, it is noticed that local Nusselt number decreases with an increase in $\mathrm{Nt}$ and $\mathrm{Nb}$. The local Sherwood number decreases with an increase in $\mathrm{Nt}$, whereas it increases with an increase in $\mathrm{Nb}$.

To verify the accuracy of the numerical results, we compared our results with those reported by Akbar et.al. [24] and Malik et.al. [25] (for regular fluids) as shown in table 1. The results are very good agreement, thus lending confidence to the accuracy of the present results.

\section{CONCLUSIONS}

We inspected the feature of radiative powell-Eyring nano fluid past a stretching sheet with variable thickness and viscous dissipation. An increment in wall thickness parameter results in decrease of velocity, temperature and concentration profiles. Enhancement in the fluid temperature is observed for higher thermophoresis parameter. Temperature profile increases for increasing values of radiation and viscous dissipation. Magnitude of both local Nusselt number and Sherwood numbers are decreasing functions of $N t$.

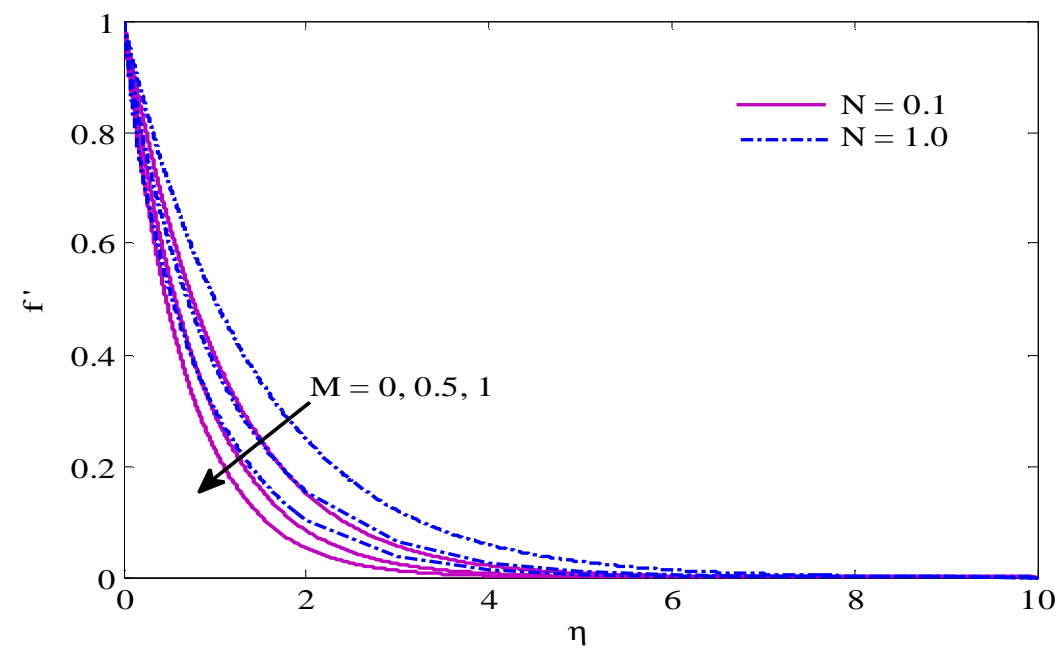

Figure 2: Velocity Profiles for Various Values of $M \& N$. 


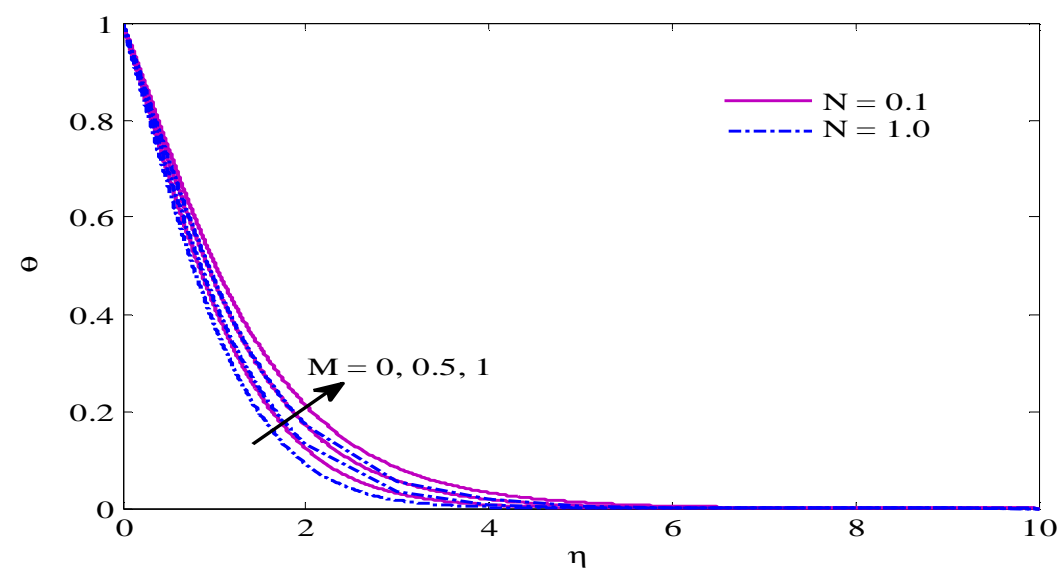

Figure 3: Temperature Profile for Various Values of $M \& N$.

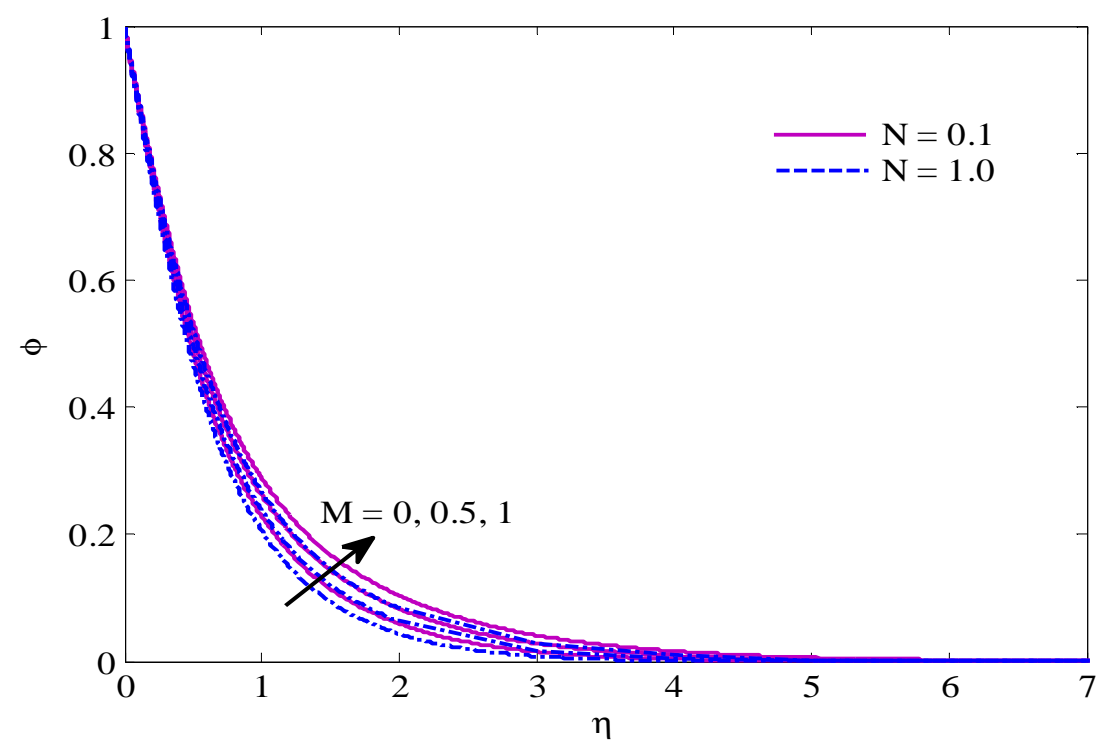

Figure 4: Concentration Profiles for Various Values of $M \& N$.

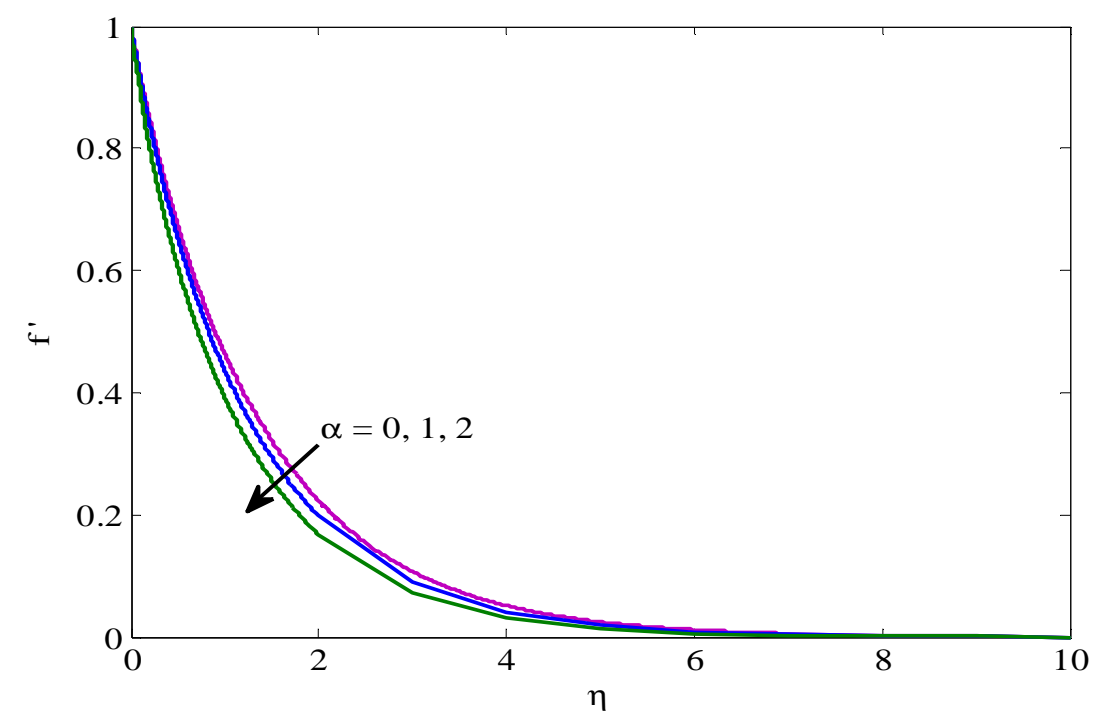

Figure 5: Velocity Profile for Various Values of $\alpha$ 


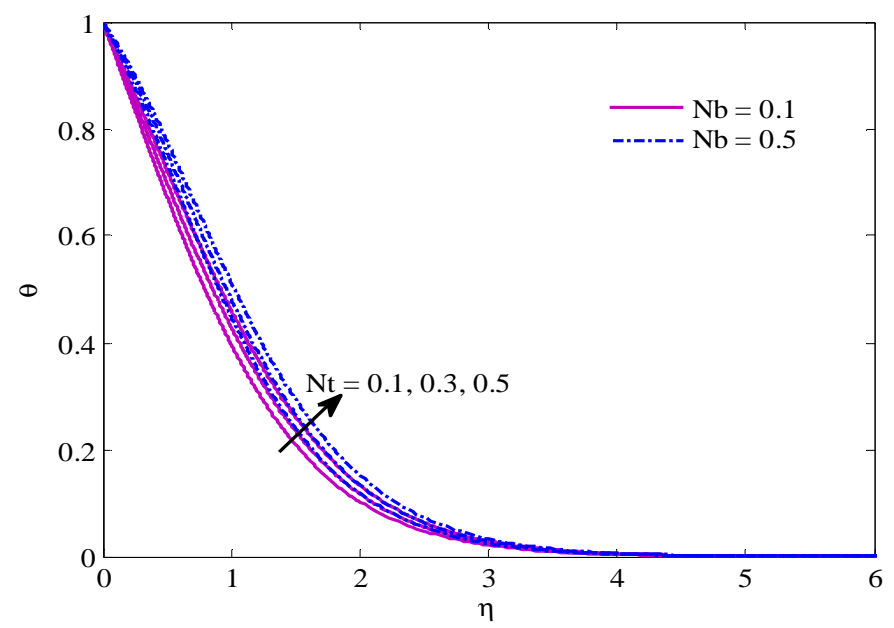

Figure 6: Temperature Profiles for Various Values of $N t \& N b$.

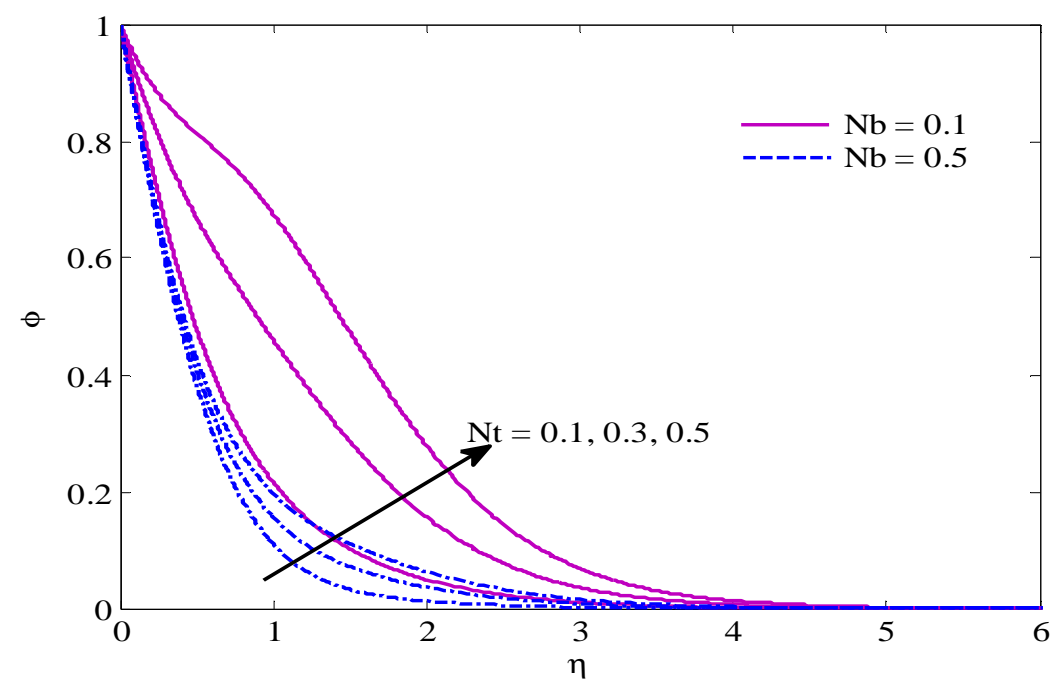

Figure 7: Concentration Profiles for Various Values of $N t \& N b$.

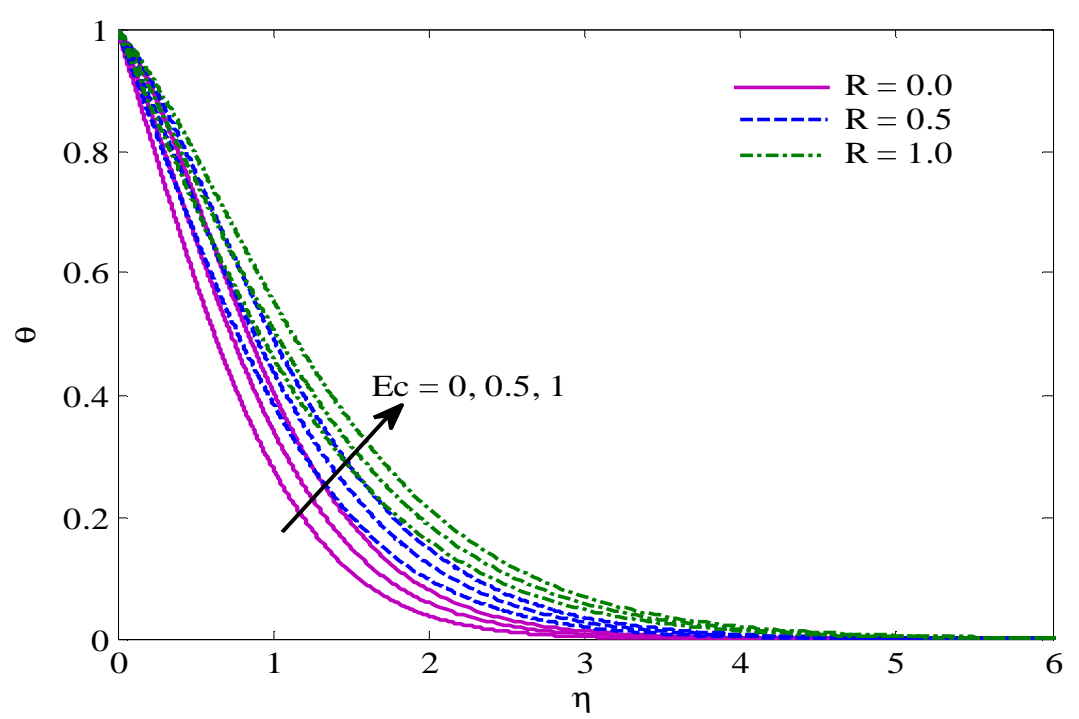

Figure 8: Temperature Profile for Various Values of $E c \& R$. 


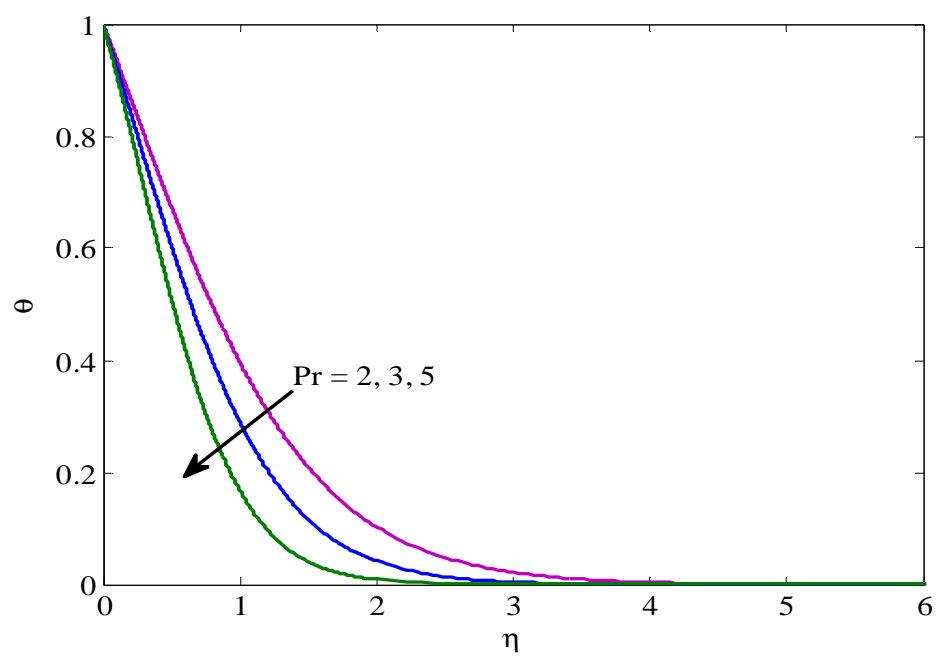

Figure 9: Temperature Profiles for Various Values of $\operatorname{Pr}$.

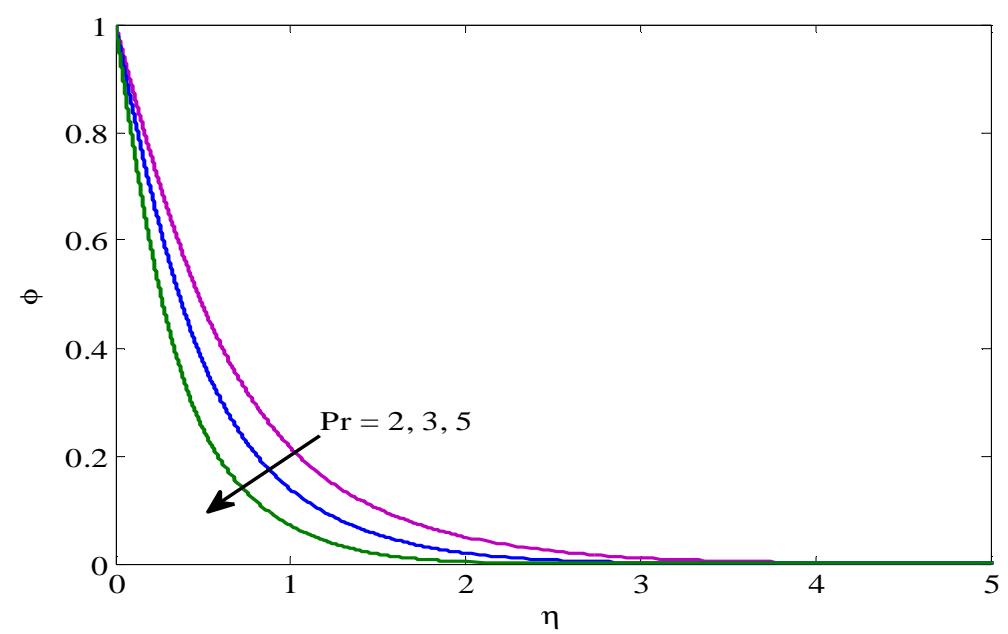

Figure 10: Concentration Profiles for Various Values of $\operatorname{Pr}$.

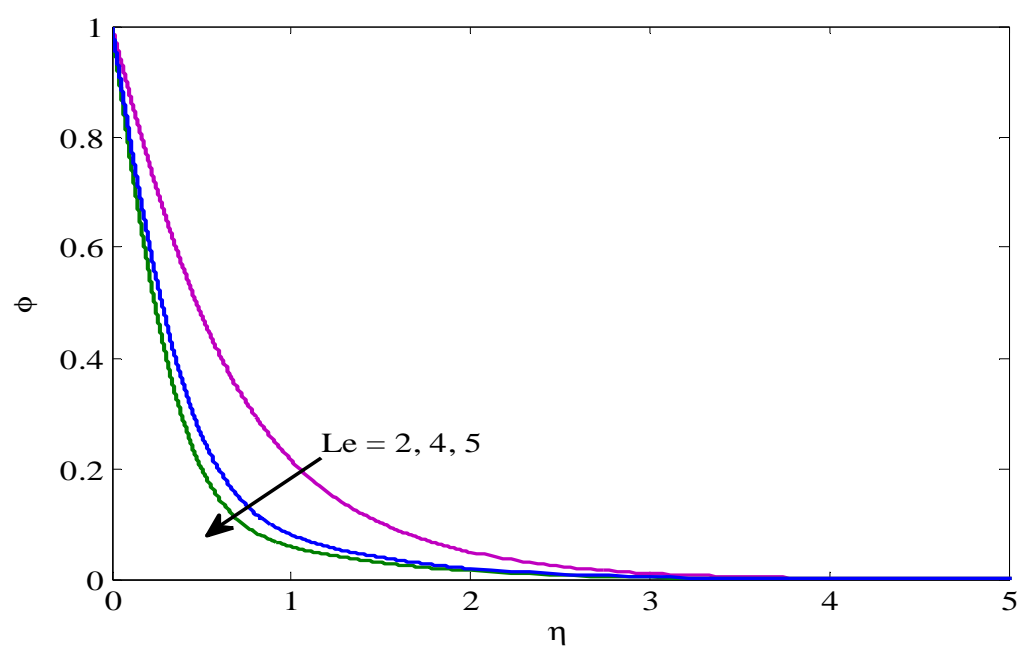

Figure 11: Concentration Profiles for Various Values of $L e$. 


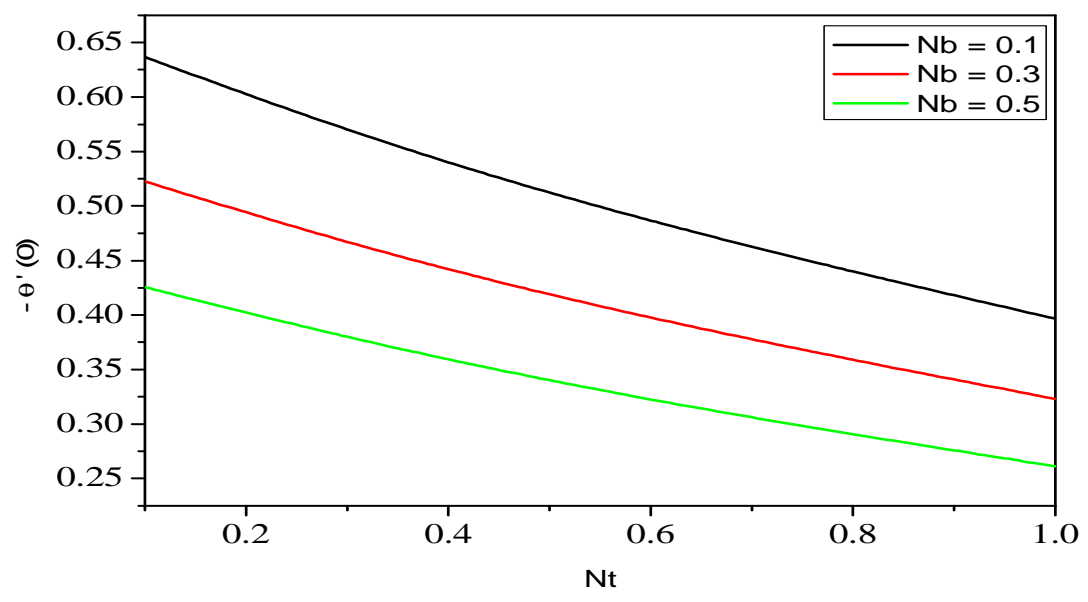

Figure 12: Local Nusselt number for various values of $N t \& N b$.

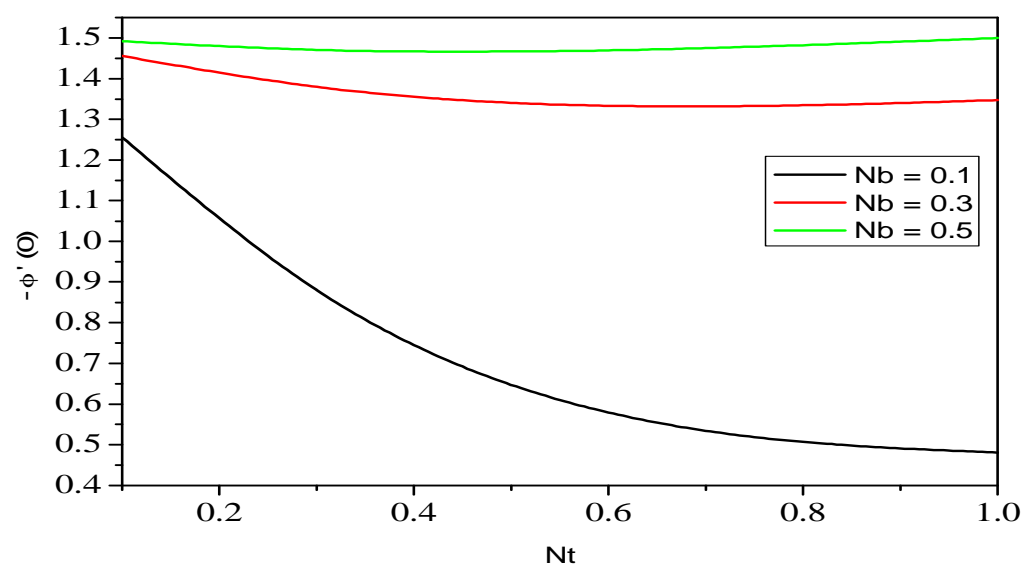

Figure 13: Local Sherwood Number for Various Values of $N t \& N b$.

Table 1: Comparison of Skin-Friction Coefficient $C_{f} \operatorname{Re}^{1 / 2}$ with the Available

Results in Literature for different Values of $M$ when $n=\lambda=\alpha=0$

\begin{tabular}{|c|c|c|c|}
\hline \multicolumn{3}{|c|}{$C_{f} \mathrm{Re}^{1 / 2}$} & \\
\hline$M$ & Present Study & Akbar et al. [24] & Malik et al.[25] \\
\hline 0 & 1 & 1 & 1 \\
0.5 & -1.118034 & -1.11803 & -1.11802 \\
1 & -1.414214 & -1.41421 & -1.41419 \\
5 & -2.449490 & -2.44949 & -2.44945 \\
10 & -3.316625 & -3.31663 & -3.31657 \\
100 & -10.049874 & -10.04988 & -10.04981 \\
500 & -22.383029 & -22.38303 & -22.38294 \\
1000 & -31.638584 & -31.63859 & -31.63851 \\
\hline
\end{tabular}

\section{REFERENCES}

1. Bilal Ashraf M., Hayat T., and Alsaedi A., (2015), Three-dimensional flow of Eyring-Powell nanofluid by convectively heated exponentially stretching sheet, Eur. Phys. J. Plus., Vol. 130(5), DOI 10.1140/epjp/i201515005-9. 
2. Hayat T., Makhdoom S., Awais M., Saleem S., and Rashidi M.M, (2016), Axisymmetric Powell-Eyring fluid flow with convective boundary condition: optimal analysis, Appl. Math. Mech. -Engl. Ed., Vol. 37(7), pp.919-928.

3. Javed T, Ali N, Abbas Z and Sajid M, (2013), Flow of an Eyring-Powell Non-Newtonian Fluid over a Stretching Sheet, Chem. Eng. Comm., 200:327-336, 2013.

4. Hayat T., Ullah I, Alsaedi A., and Farooq M., (2017), MHD flow of Powell-Eyring nanofluid over a non-linear stretching sheet with variable thickness, Results in Physics, Vol.7, pp.189-196.

5. Nazar R., Tham L., Pop I., and Ingham D.B., (2011), Mixed Convection Boundary Layer Flow from a Horizontal Circular Cylinder Embedded in a Porous Medium Filled with a Nanofluid, Transp Porous Med., Vol. 86, pp.517536

6. Hunt A.J., (1978), Small particle heat exchangers, J Renew Sustain Energy Lawrence Berkeley Lab Report Number LBL-7841.

7. Masuda H., Ebata A., Teramae K., and Hishinuma N., (1993), Alteration of thermal conductivity and viscosity of liquid by dispersing ultra-fine particles (dispersion of c-A12O3, $\mathrm{SiO} 2$ and $\mathrm{TiO} 2$ ultra-fine particles), Netsu Bussei (in Japanese), Vol.4, pp.227- 233.

8. Choi S.U.S., and Eastman J.A., (1995), Enhancing thermal conductivity of fluids with nanoparticles. in: The Proceedings of the 1995 ASME International Mechanical Engineering Congress and Exposition, San Francisco, USA, ASME, FED 231/ MD 66: 99-105.

9. Buongiorno J., (2006), Convective transport in nanofluids, ASME J Heat Transf., Vol.128, pp.240-250.

10. Batchelor G.K., (1976), Brownian diffusion of particles with hydrodynamic interaction, J Fluid Mech., Vol.74, pp.1-29.

11. Walker K.L., Homsy G.M., and Geyling F.T., (1979), Thermophoretic deposition of small particles in laminar tube flow, J Coll Interf Sci., Vol. 69, pp.138-147.

12. Pratsinis S.T., and Kim K.S., (1989), Particle coagulation, diffusion and thermophoresis in laminar tube flows, J Aeros Sci., Vol.20, pp.101-111.

13. Otanicar T.P., and Golden J.S., (2009), Comparative environmental and economic analysis of conventional and nanofluid solar hot water technologies, Environ Sci \& Tech., Vol. 43, pp.6082-6087.

14. Tyagi H., Phelan P., and Prasher R., (2009), Predicted efficiency of a low-temperature nanofluid-based direct absorption solar collector, J Solar Ener Eng., Vol.131, Article ID:041004.

15. Buongiorno J., and Hu L.W., (2009), Nanofluid heat transfer enhancement for nuclear reactor application. Proceedings of the ASME 2009 2nd Micro/Nanoscale Heat \& Mass Transfer International Conference, MNHMT 2009. DOI: 10.1115/ MNHMT2009-18062.

16. Huminic G., and Huminic A., (2012), Application of nanofluids in heat exchangers: A review, Renew Sust Ener Reviews, Vol. 16, pp.5625-5638.

17. Ramzan M., Bilal M., and Chung J.D., (2017), Radiative Flow of Powell-Eyring Magneto-Nanofluid over a 
Stretching Cylinder with Chemical Reaction and Double Stratification near a Stagnation Point, PLoS ONE, Vol.12(1): e0170790. DOI:10.1371/journal. pone.0170790.

18. Ullah I., Khan I., and Shafie S., (2016), MHD Natural Convection Flow of Casson Nanofluid over Nonlinearly Stretching Sheet Through Porous Medium with Chemical Reaction and Thermal Radiation, Nanoscale Research Letters, Vol.11:527.

19. Mustafa M., Mushtaq A., Hayat T., and Ahmad B., (2014), Nonlinear Radiation Heat Transfer Effects in the Natural Convective Boundary Layer Flow of Nanofluid Past a Vertical Plate: A Numerical Study, PLoS ONE, Vol.9(9): e103946. DOI:10.1371/journal.pone.0103946.

20. Gireesha B.J., Venkatesh P., Shashikumar N.S., and Prasannakumara B.C., (2017), Boundary layer flow of dusty fluid over a radiating stretching surface embedded in a thermally stratified porous medium in the presence of uniform heat source, Nonlinear Engineering, DOI 10.1515/nleng-2016-0058.

21. Ramzan M., Bilal M., Chung J.D., and Mann A.B., (2017), On MHD radiative Jeffery nanofluid flow with convective heat and mass boundary conditions, Neural Comput \& Applic., pp.1-10, DOI 10.1007/s00521-0172852-8

22. Vajravelu K., and Hadjinicolaou A., (1993), Heat transfer in a viscous fluid over a stretching sheet with viscous dissipation and internal heat generation, Int Commun Heat Mass Transfer, Vol.20, pp.417-430.

23. Ralston A., and Wilf H.S., (Eds.) (1976), Mathematical methods for digital computers, Vol. 1, John Wiley \& Sons

24. Akbar N.S., Nadeem S., Haq R.U., and Khan Z.H., (2013), Numerical solutions of Magnetohydrodynamic boundary layer flow of tangent hyperbolic fluid towards a stretching sheet, Indian Journal of Physics, Vol.87(11), pp.1121-1124.

25. Malik M.Y., Salahuddin T., Arif Hussain, and Bilal S., (2015), MHD flow of tangent hyperbolic fluid over a stretching cylinder : Using Keller box method, Journal of Magnetism and Magnetic Materials, Vol.395, pp.271276. 